\title{
Optical Image-guided Surgery-Where Do We Stand?
}

Stijn Keereweer, ${ }^{1,2}$ Jeroen D. F. Kerrebijn, ${ }^{1}$ Pieter B. A. A. van Driel, ${ }^{2}$ Bangwen Xie, ${ }^{2}$ Eric L. Kaijzel, ${ }^{2}$ Thomas J. A. Snoeks, ${ }^{2}$ Ivo Que, ${ }^{2}$ Merlijn Hutteman, ${ }^{3}$ Joost R. van der Vorst, ${ }^{3}$ J. Sven D. Mieog, ${ }^{3}$ Alexander L. Vahrmeijer, ${ }^{3}$ Cornelis J. H. van de Velde, ${ }^{3}$ Robert J. Baatenburg de Jong, ${ }^{1}$ Clemens W. G. M. Löwik ${ }^{2}$

${ }^{1}$ Department of Otorhinolaryngology, Head \& Neck Surgery, Erasmus Medical Center, P.O. Box 2040, 3000 CA, Rotterdam, The Netherlands

${ }^{2}$ Department of Endocrinology, Leiden University Medical Center, Leiden, The Netherlands

${ }^{3}$ Department of Surgery, Leiden University Medical Center, Leiden, The Netherlands

\begin{abstract}
In cancer surgery, intra-operative assessment of the tumor-free margin, which is critical for the prognosis of the patient, relies on the visual appearance and palpation of the tumor. Optical imaging techniques provide real-time visualization of the tumor, warranting intra-operative image-guided surgery. Within this field, imaging in the near-infrared light spectrum offers two essential advantages: increased tissue penetration of light and an increased signal-tobackground-ratio of contrast agents. In this article, we review the various techniques, contrast agents, and camera systems that are currently used for image-guided surgery. Furthermore, we provide an overview of the wide range of molecular contrast agents targeting specific hallmarks of cancer and we describe perspectives on its future use in cancer surgery.
\end{abstract}

Key words: Optical imaging, Image-guided surgery, Molecular imaging, Near-infrared fluorescence

\section{Introduction}

$\mathrm{O}$ ver the last decades, imaging technologies have made significant developments, resulting in their current perpetual role in clinical oncology. The field has expanded greatly and now comprises various modalities, including ultrasonography, computed tomography (CT), magnetic resonance imaging (MRI), positron emission tomography (PET), and single-photon-emission computed tomography. Because each modality has its specific advantages and disadvantages, the combination of different techniques has become standard practice for tumor detection, staging, and treatment evaluation [1].

However, when surgery is required, translation of these images to the operation field remains a major challenge for the surgeon. Visual appearance and palpation is the only

Correspondence to: Stijn Keereweer; e-mail: s.keereweer@erasmusmc.nl way to discriminate between tumor and normal tissue and consequently determine an adequate tumor-free margin during surgery. This is a critical aspect of oncologic surgery because, apart from tumor characteristics (i.e., stage, differentiation grade), complete surgical removal is pivotal for the prognosis of the patient.

Real-time imaging technologies could offer the possibility to put the images right under the hands of the surgeon, warranting intra-operative image-guided surgery. In order to detect malignant cells or tissues, the various hallmarks of cancer can be used as a target for imaging strategies: increased growth and growth factor signaling receptors, limitless replicative potential, sustained angiogenesis, and increased proteolytic activity resulting in tissue invasion and metastasis (Fig. 1) [2].

The characteristic of increased metabolism is exploited in PET technology, using the glucose-mimetic 2-deoxy-2- $\left[{ }^{18} \mathrm{~F}\right]$ fluoro-D-glucose. Tumor cells most frequently use glycol- 


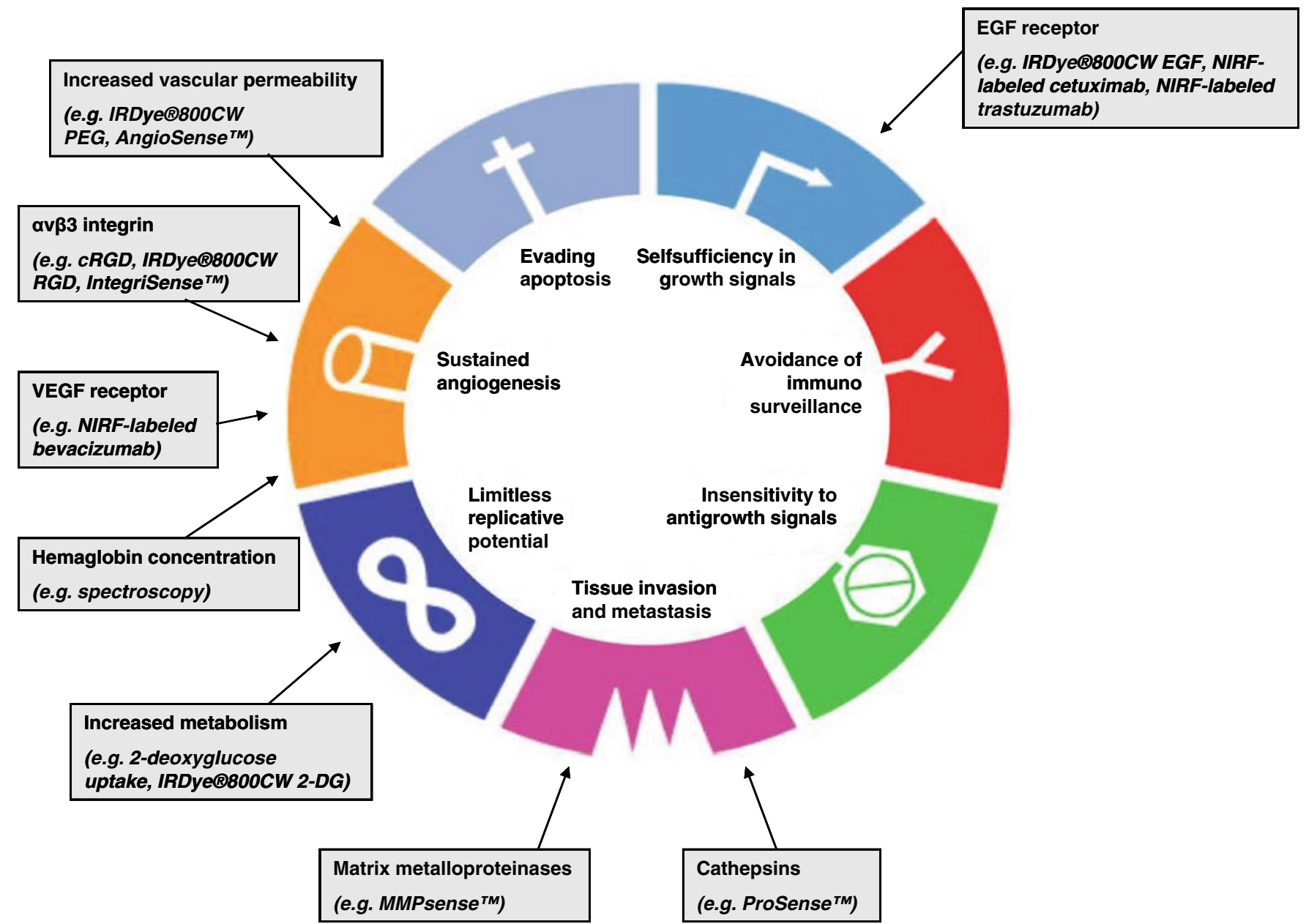

Fig. 1. Hallmarks of cancer and their targets for optical imaging. EGF epidermal growth factor, $c R G D$ cyclic arginine-glycineaspartate, VEGF vascular endothelial growth factor, NIRF near-infrared fluorescence. Edited from with kind permission from Kroemer, G \& Pouyssegur, J; Tumor cell metabolism: cancer's Achilles' heel. Cancer Cell. 2008 Jun;13(6):472-82.

ysis for energy generation, resulting in an increased glucose metabolism and the overexpression of glucose transporters (GLUTs) [3]. 2-Deoxy-2-[ $\left.{ }^{18} \mathrm{~F}\right]$ fluoro-D-glucose is taken up by metabolically active cells, making it applicable in a wide range of tumors [4]. For intra-operative evaluation of tumor localization and margin status in breast cancer surgery, a hand-held PET probe to detect the high-energy gamma rays during surgery has been developed [5].

Although very useful for diagnostic applications, the PET imaging technology has several disadvantages for widely used intra-operative practice. Due to its limited spatial resolution, small tumors $(<1 \mathrm{~cm})$ have proven difficult to detect by this hand-held probe [5]. Furthermore, the use of PET technology is restricted to specialized centers for logistical, practical, and financial reasons. Radiochemists, a cyclotron, and specialized waste processing facilities are required in order to produce the radiopharmaceuticals. Although radiation exposure for the patient and operation personnel is minimized due to tracer amounts of the very short-lived agents, the short half-life time limits the time frame in which the agents have to be administered. This requires careful planning with the risk of high expenses when an operation procedure is delayed.
Interesting developments have been made in the last years using Raman spectroscopy. A Raman spectrum is generated by a shift in frequency in the incident excitation light of tissue, resulting in in-elastic scattering. The shift in frequency is caused by discrete changes in emergent light, above and below the wavelength of the incident photons, due to the vibrational frequencies of the bio-molecules that constitute the tissue. There are several characteristic features of spectroscopy which make it attractive for oncological applications. Specific lesions can be identified using optical fibers for in vivo measurements. Furthermore, they do not require the use of dyes, labels, or other contrast-enhancing agents, implicating that tissues can be studied in their native states.

Various groups have performed Raman spectroscopic studies where spectral differences have been reported between normal and cancerous lesions. In this way, the detection of cancers and precancers has been described in skin, mucosal surfaces, and solid organs [6-8].

These interesting results suggest its relevance to clinical oncological applications. However, several hurdles have yet to be overcome. The Raman effect comprises a very small fraction $\left(\sim 1\right.$ in $\left.10^{7}\right)$ of the incident photons, resulting in 
much weaker signals than autofluorescence. Hence, these signals can be easily masked by the broad-band fluorescence background, hampering interpretation of the spectra. Additionally, in most of these studies, a fiber-optic Raman probe is required to access the tissues of interest in vivo. Although a necessary and important step towards a widespread clinical use, such fiber-based Raman technology using manageable probes with real-time translation software is yet to be developed [9].

In the light of image-guided surgery, a significant amount of work has been done in the field of optical imaging. In optical imaging, the properties of light emitted from a light source (e.g., laser, LED, Xenon) are exploited to image anatomic or chemical characteristics of tissue. Imaging of optical contrast can be performed using either the properties intrinsic to the tissue, or, analogous to many radiolabeled agents, using ligands conjugated to an optically active reporter to target a recognized disease biomarker [1]. In this way, optical imaging could provide a valuable addition to the current modalities of image-guided surgery. This review outlines the various techniques, contrast agents, and camera systems that are currently used for image-guided surgery. Furthermore, an overview of the wide range of molecular contrast agents that each target specific hallmarks of cancer is provided and the perspectives on its future use in cancer surgery are described.

\section{Autofluorescence}

Autofluorescence refers to the intrinsic fluorescence of the tissue that is excited when activated by ultraviolet, visible, or near-infrared (NIR) radiation of suitable wavelength. Because cancerous transformation leads to morphologic and biochemical alterations, which affect the optical properties of the tissue, in some cases, the autofluorescence may actually illuminate the structures of interest and can serve as a useful diagnostic indicator [10]. This holds a significant advantage, for there is no exogenous agent required which could complicate regulatory approval for clinical use.

Using this technique, dysplastic and malignant tissues have been shown to excite increased red fluorescence due to an increase of endogenous porphyrins in their tissues and decreased green fluorescence because of breakdown of collagen cross links within the superficial stroma. Both increase in the red/green fluorescence ratio and an increase in $\mathrm{NAD}(\mathrm{P}) \mathrm{H}$ fluorescence are reported to be predictors of dysplasia and malignancy [11-14].

Two-photon fluorescence microscopy is a relatively novel technique to study cell biology in vitro and in vivo. This technology, based on the quasi-simultaneous absorption of two or more photons, requires less signal intensity, increases penetration depth to several millimeters and offers better axial resolution compared to one-photon fluorescence techniques. It has been used for dynamic imaging of tumor growth and invasion in vivo using a modified skin-fold chamber for orthotopic implantation [15]. To our knowledge, no intra-operative applications of this technique have been described.

However, despite the initial optimism, the determination of the various mechanisms that influence the signal has proven to be a complex process [16]. These studies clearly indicate a robust correlation between optical signature and the phase of the disease progression but as long as the relative contributions of the individual components are not isolated, the specificity of the tumor detection remains difficult to determine.

\section{Optical Imaging with Conventional Fluorescence}

Optical imaging revolves around the concept of signal-tobackground ratio (SBR), which in cancer imaging is usually referred to as the tumor-to-background ratio. To detect the targeted (tumor) cells, the tumor-specific signal must be significantly discriminated from the nonspecific surrounding signals.

The conventional fluorescent techniques use probes in the visible light spectrum $(\sim 400-600 \mathrm{~nm})$, which is not optimal for intra-operative image-guided surgery. This spectrum is associated with a relatively high level of nonspecific background light, resulting in a low SBR. In addition, the absorption of visible light by biological chromophores, in particular hemoglobin, limits the penetration depth to a few millimeters.

\section{Optical Imaging with Near-infrared Fluorescence}

Over the last decade, the development of NIR fluorophores and nanomaterials has led to a revolution in optical imaging. The concept of using NIR light has proven a crucial step towards its application in intra-operative image-guided surgery.

The combination of light absorption by hemoglobin in the visible light spectrum $(<600 \mathrm{~nm})$ and other components (e.g., water and lipids) in the infrared range ( $>900 \mathrm{~nm})$, offers an optical imaging window from approximately $650-900 \mathrm{~nm}$ in which the absorption coefficient of tissue is at a minimum. Consequently, light scattering and autofluorescence are decreased and tissue penetration is increased in this spectrum, stimulating the development of a range of probes that provide a high SBR.

\section{Near-infrared Spectroscopy}

The intrinsic optical absorption signals of blood, water, and lipid, correlated with increased hemoglobin concentration due to angiogenesis and decreased hemoglobin saturation due to hypermetabolism, can be utilized in NIR spectroscopy to detect and localize cancer [17]. Using three-dimensional parallel-plate diffuse optical tomography [18] or time-domain optical mammography (Softscan ${ }^{\circledR}$, Advanced Research Technologies, 
Montreal, Canada) to measure photon migration through the breast, variations in the functional and structural NIR properties (e.g., scattering, oxy-, and deoxy-hemoglobin concentrations) were observed, enabling differentiation between benign and [19] malignant tumors. However, these techniques are not directly suitable for intra-operative use.

\section{Non-targeted Exogenous Contrast Agents}

Various nonspecific agents have been developed that are currently available for research and clinical use (e.g., fluorescein, indocyanine green (ICG), cresyl violet acetate, toluidine blue, Lugol's iodine). ICG (emission peak, $830 \mathrm{~nm}$ ) has optical properties that are suitable for NIR imaging, which have been exploited for imaging of angiogenesis and identification of hepatic segments for facilitation of hepatic resection [20,21]. Moreover, promising results have been reported in the field of sentinel lymph node mapping, which we will describe later. In principle, because these agents are not tumor specific, they are not optimally suited for determination of the tumor-free margin. Very recently however, real-time demarcation of small and grossly unidentifiable liver cancers using ICG has been described due to the disordered biliary excretion of ICG in cancer tissues and noncancerous liver tissues compressed by the tumor [22].

\section{Non-targeted Activatable Organic Fluorophores}

When focusing on the expansive character of the tumor resulting from up-regulation of proteolytic enzymes, a different strategy can be followed by using activatable fluorophores. This method allows detection of proteases that are relatively abundant in malignant tissue, which can be associated with specific characteristics (e.g., invasive, aggressive, or metastatic tendency) of the tumor. These agents are injected in a quenched (i.e., inactivated) state, resulting in minimal fluorescence at the time of adminis- tration. When cleaved by specific enzymes, the agent becomes dequenched (i.e., activated), and fluorescence can be measured. Due to the cleavability, these probes have higher SBR when compared to nonspecific probes.

Weissleder et al. have developed a series of such cleavable NIR probes that are activated by proteases such as cathepsins and matrix metalloproteinases $[23,24]$ that are currently commercially available (Visen Medical, Boston, MA, USA). A different quencher mechanism, using an inhibitory domain made up of negatively charged residues fused to activatable polyarginine-based cell-penetrating peptides has been described by Tsien et al. [25] for use in the detection of matrix metalloproteinases. However, these agents are not targeting specific molecules in cancer cells only. Cathepsins and matrix metalloproteinases are also produced by macrophages and neutrophils, and hence are abundant in inflammatory tissue [26] and in cerebral ischemia [27]. Nonetheless, our preclinical results in rats (Mieog et al., in press) and the previously mentioned studies are very promising (Fig. 2).

\section{Targeted Organic Fluorophores}

Essential for the limitless replicative potential of tumor cells are the increased metabolism and expression of growth signaling receptors, combined with increased tumor angiogenesis to supply sufficient oxygen and nutrients. These features can be exploited by targeted NIR fluorescent agents, which provide molecularly specific detection of cancer cells (Fig. 1). In these agents, the NIR fluorophore has been conjugated to a specific targeting ligand or monoclonal antibody. Such agents have shown excellent SBR in whole animals and images can be collected over longer periods than the short-lived radiotracers labeled with fluorine-18 or carbon-11. For increased growth factor receptor expression, several groups have reported the use of various monoclonal antibodies coupled to fluorophores for imaging of the epidermal growth factor (EGF) receptor, Her2/ neu receptor, or vascular endothelial growth factor (VEGF) receptor in all kinds of different tumors [28-30]. In these
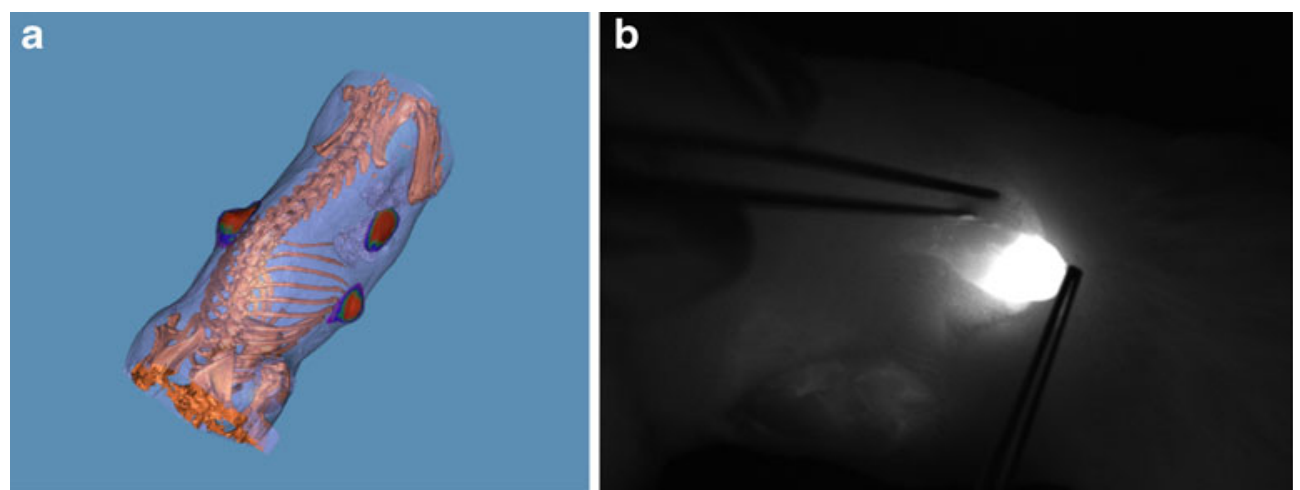

Fig. 2. Detection of breast cancer using the non-targeted activatable organic NIRF probe ProSense $680^{\mathrm{TM}}$ in a syngeneic rat model of primary breast cancer. a 3-Dimensional fusion of contrast-enhanced micro-CT and fluorescence imaging. In collaboration with J. Dijkstra, Division of Image Processing, LUMC. b NIRF image-guided resection of one of the primary tumors using the Fluobeam ${ }^{\mathrm{TM}}$. 
studies, the fluorophores Cy 5.5 (excitation $678 \mathrm{~nm}$, emission $703 \mathrm{~nm}$ ) and Alexa Fluor 750 (excitation 749 nm, emission $775 \mathrm{~nm}$ ) were used.

An exciting new approach, similar to the non-targeted activatable organic fluorophores described earlier, is the use of quenched fluorophores conjugated to tumor-targeting monoclonal antibodies. This approach was successfully explored by Kobayashi and et al. [31]. Kobayashi et al. used avidin (targeting the d-galactose receptor) and trastuzumab (HER2 mAb) labeled with the TAMRA (fluorophore)-QSY7 (quencher) pair. After internalization into the cell, the probe was cleaved, resulting in dequenching and target-specific fluorescence imaging with high SBR.

A fluorophore bound to a specific ligand can be internalized and its uptake can be monitored, as was illustrated with VEGF and EGF $[32,33]$. The latter was conjugated to the IRdye $800 \mathrm{CW}$ (Li-Cor Bioscience, Lincoln, NE, USA), a fluorophore with a peak excitation at $785 \mathrm{~nm}$ and emission at $810 \mathrm{~nm}$. Due to the higher excitation frequency, IRdye $800 \mathrm{CW}$ has even better tissue penetration and SBR than Cy 5.5 [34].

In strategies for imaging (tumor) angiogenesis, a crucial concept has been the targeting of alpha-v-beta-3 $\left(\alpha_{v} \beta_{3}\right)$ integrin, a critically important adhesion molecule in the regulation of angiogenesis. This molecule can be found at the sprouting ends of newly formed blood vessels but also frequently on many epithelial tumor cells. High expression of adhesion receptors can be detected when targeting $\alpha_{\mathrm{v}} \beta_{3}$ integrin by cyclic arginine-glycine-aspartate (cRGD) conjugated to Cy 5.5 or IRdye $800 \mathrm{CW}$ [35]. The use of the quenching technique has been described in this respect as well, by means of a quenched cRGD molecule (RAFTc(-RGDfK-)(4)-Cy5-SS-Q), which becomes activated upon internalization into the cell [36].

Increased $\alpha_{v} \beta_{3}$ expression can also be visualized by direct binding of a small peptidomimetic antagonist coupled to a NIR fluorescent dye (VivoTag-S680), known as IntegriSense (Visen Medical, Boston, MA, USA). IntegriSense has a much higher specificity for $\alpha_{v} \beta_{3}$ integrin compared to RGD-based probes. It was shown to co-localize at the surface of both $\alpha_{v} \beta_{3}$ integrin positive endothelial and tumor cells. The signal is additionally enhanced by the internalization into $\alpha_{v} \beta_{3}$ integrin positive tumor cells, leading to a slower clearance of the probe from tumors compared to surrounding tissues [37]. This probe has successfully been used to visualize liver metastasis from colon cancer in a rat model. It is important to note that most fluorescent dyes are related to high background signal in the liver, kidneys, and bladder due to biodistribution and clearance of the agent, whereas in this study, the liver metastases could be clearly demarcated (Mieog \& Hutteman et al., submitted).

In analogy with PET technology, targeting the increase in glucose metabolism due to increased expression of membrane glucose transporter proteins (i.e., GLUT) is a promising strategy. Very interesting results have recently been published regarding imaging of glucose uptake, as reflected by increased GLUT, in intracranial gliomas using the glucose analog 2-deoxyglucose conjugated with IRDye800CW [38].

\section{Nanoparticles}

Quantum dot nanoparticles are small crystals $(2-10 \mathrm{~nm}$ diameter), made of inorganic semiconductor materials. They possess several physical properties that make them appealing for use as imaging reporters. The high quantum yields result in high signal intensity, enabling detection at lower concentrations compared to organic fluorophores. In addition, the fluorescence emission spectra can be tuned, depending on their size, allowing for multiplexed imaging. Furthermore, quantum dot nanoparticles have proven to be photostable and have the possibility to target multiple biomarkers, due to their ability to contain multiple probe molecules. Finally, multimodal targeted quantum dot-based nanoparticles coated with paramagnetic micellar shells have been described, allowing for both optical and magnetic resonance detection of tumor angiogenesis (Fig. 3) [39].

However, the toxicity of quantum dot nanoparticles is a serious concern. The problem is that most quantum dots (albeit a very diverse group of substances) are based on heavy metal cores (e.g., Cd-Se, Cd-Te), which have been reported to be cytotoxic in their soluble form due to the release of toxic $\mathrm{Cd}^{2+}$ ions and their surface chemistry and stability towards aggregation [40, 41]. These issues raise such significant hazards that, at present, clinical application of quantum dot nanoparticles does not seem feasible.

Silica nanoparticles have been developed as an alternative to quantum dots that combine the versatility and functionality of organic dyes with the stability and biocompatibility of the silica surface [42]. In a recent study, the biodistribution, including long-term quantitative tissue distribution, subcellular distribution, and the toxicity of silica nanoparticles were assessed in a mouse model. The results indicated that the small size of the silica nanoparticles resulted in high permeability and lengthy accumulation of the agent in lungs, liver, and spleen and could potentially cause liver injury when intravenously injected [43]. Because size, surface area, surface chemistry, solubility, and shape are probably all key features that play a role in determining the harmful potential for engineered nanomaterials [44], extensive research will have to be performed over the coming years in order to address these issues.

In conclusion, several strategies are at hand in the optical imaging field using NIR with probes that can reach significant SBR and can be combined with targeting ligands to increase the specificity and sensitivity of tumor detection (Fig. 4). Because organic fluorophores are more biocompatible than inorganic agents, it is expected that, when using the former agents, toxicity issues will play a minor role, as illustrated by ICG and fluorescein which are already widely used in the clinic. However, for clinical translation of these results, pharmacokinetic studies are required for each fluorophore or fluorophore conjugate. 

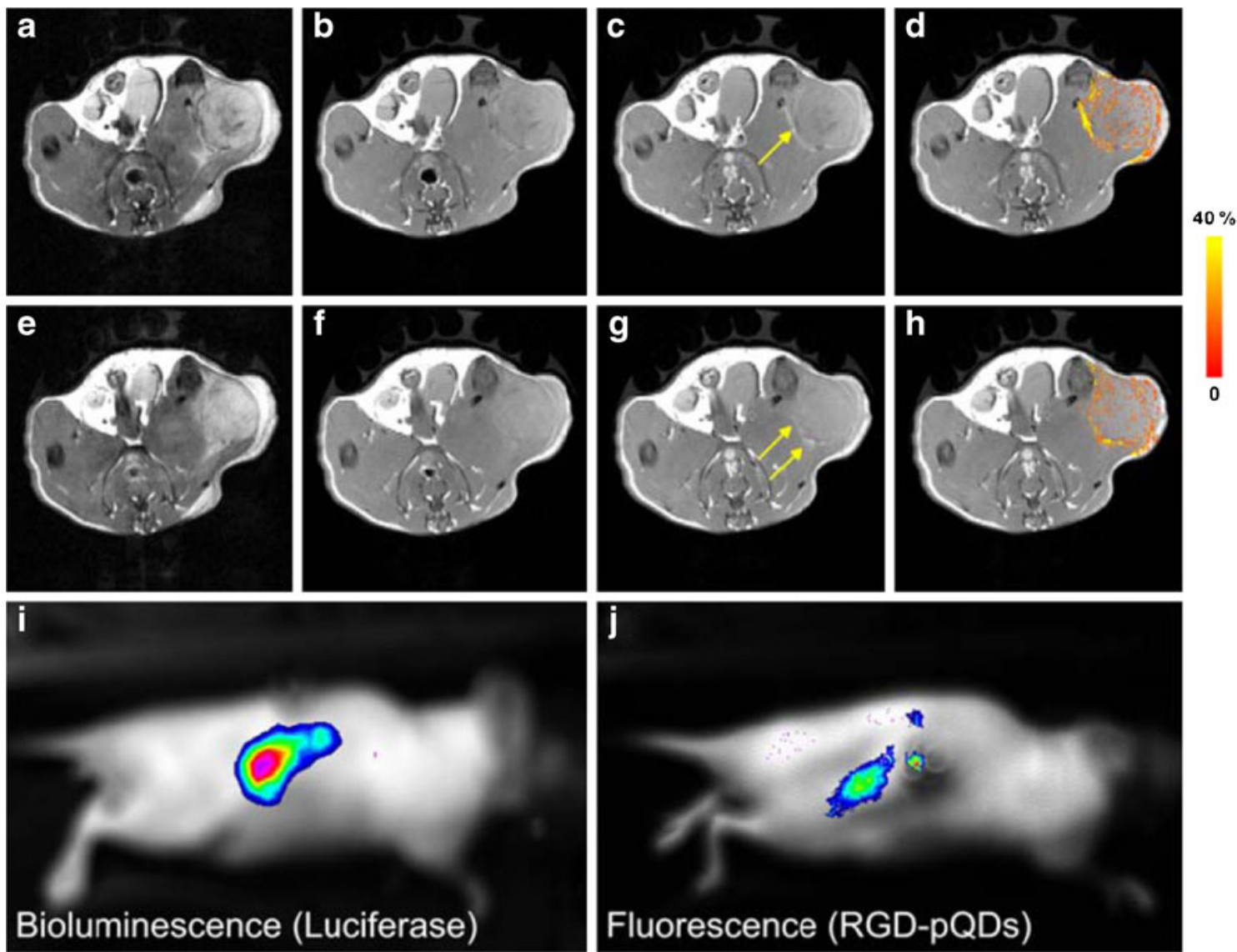

Fig. 3. Magnetic resonance and optical molecular imaging of tumor angiogenesis using alpha-v-beta-3 targeted multimodal quantum dot-based nanoparticles. T2-weighted images (a, e), collected before the nanoparticles were injected, show the contour of the tumor on the flank. T1-weighted images $(\mathbf{b}, \mathbf{c}, \mathbf{f}, \mathbf{g})$ with TR=800 ms were measured before (b, $\mathbf{f})$ and $45 \mathrm{~min}$ after $(\mathbf{c}, \mathbf{g})$ the injection of the nanoparticles. The arrows in $(\mathbf{c}, \mathbf{g})$ indicate bright (positive contrast) regions in the periphery of the tumor. In $\mathbf{d}, \mathbf{h}$, pixels in the tumor with signal enhancement of at least three times the noise level are color coded according to the pseudo-color scale on the right. i, $\mathbf{j}$ Bioluminescence imaging and fluorescence imaging of a Balb/c nude mouse with a luciferase-expressing renal carcinoma tumor after injection of luciferin (i). The strong bioluminescence signal is indicative of tumor growth in the right kidney. This signal colocalizes with a strong fluorescence signal (j) originating from intravenously administrated nanoparticles that are accumulated in the tumor. Republished with kind permission from Mulder WJ, Castermans $\mathrm{K}$, van Beijnum JR et al. Molecular imaging of tumor angiogenesis using alphavbeta3-integrin targeted multimodal quantum dots. Angiogenesis. 2009;12(1):17-24.

\section{Image-guided Surgery}

The properties of NIR optical imaging are clearly perfectly suited for real-time fluorescence imaging during surgery. If adequate imaging resolution can be achieved, intra-operative tumor visualization may improve radical resection without unnecessary damage to healthy tissue and has the potential to more accurately assess tumor margins during surgery. Moreover, through biochemical changes involved in the earliest stages of neoplastic development, this technique promises to identify malignant lesions before they become visible to the naked eye or currently available intra-operative imaging modalities.

Considering its wide range of applications, one could wonder why this technique has just only recently emerged. Essentially, the development has been hampered by the lack of suitable NIR probes and dedicated camera systems for the intra-operative visualization of these probes. As described above, NIR probe development has made significant progress, resulting in the urgent need for intra-operative imaging systems.

Conventional camera systems are limited to single-band NIR cameras, which have two drawbacks: they do not enable separation of autofluorescence from the probe signal and it is not possible to correct for geometric and intensity distortions caused by photon-tissue interactions. Dedicated NIR camera systems that have been used for intra-operative optical imageguided surgery include the Photodynamic Eye (PDE; Hamamatsu Photonics, Hamamatsu City, Japan) and a self-build system, the Fluorescence-assisted Resection and Exploration (FLARE ${ }^{\mathrm{TM}}$ ) [45], from the Fragioni laboratory (Brookline, MA, USA). Currently, two smaller systems have been developed: the Mini-FLARETM from the Frangioni laboratory [46] and the Fluobeam from Fluoptics (Grenoble, France; Fig. 5). 

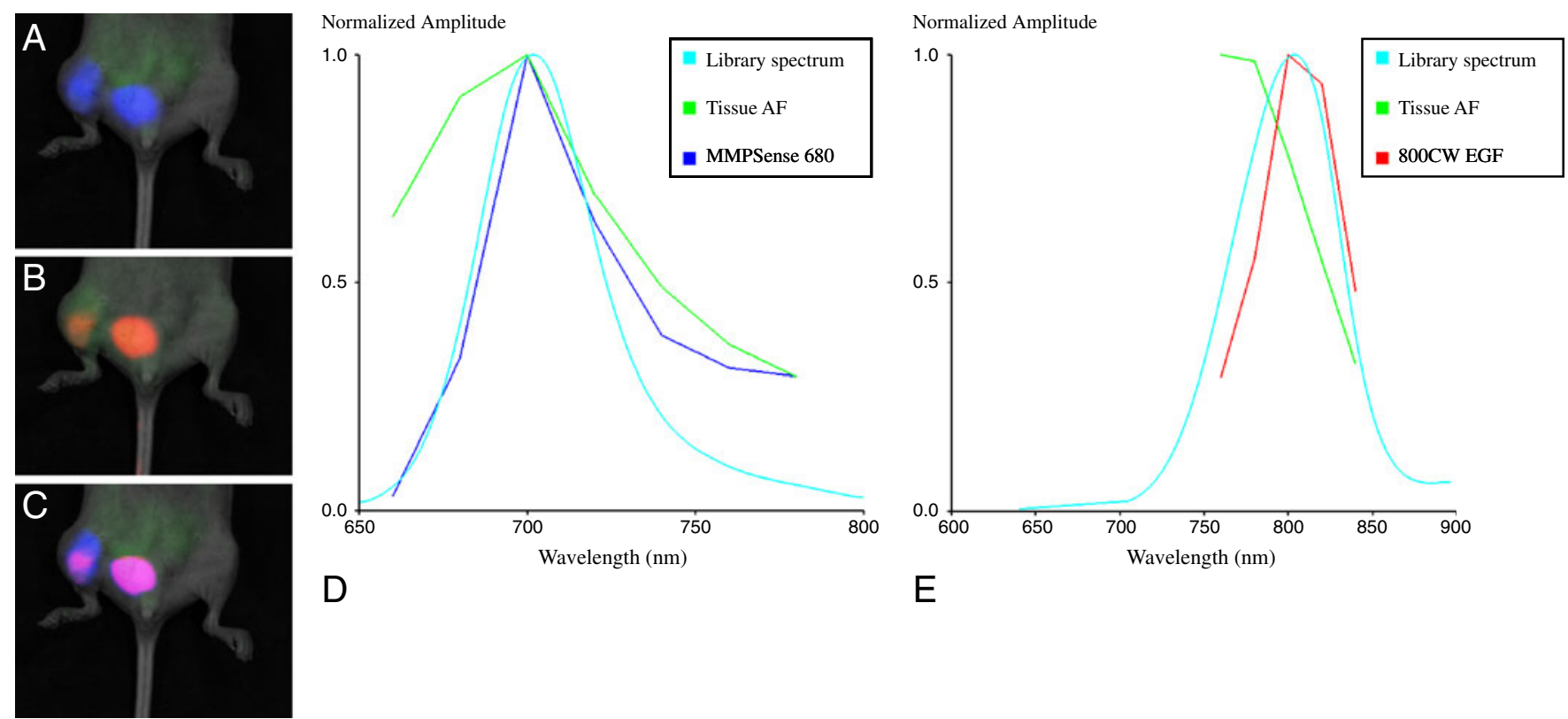

D

E

Fig. 4. Illustration of the use of two fluorescent agents with emission on different wavelengths, showing the simultaneous detection of an MDA-MB231 breast cancer bone metastases in the right hind limb of a nude mouse. MDA-MB231 cells express relatively high levels of EGFR and are highly osteolytic. Nonspecific uptake of the probes in the bladder is also shown. a Fluorescent image showing the blue signal of MMPSense ${ }^{T M}$ 680; green is tissue autofluorescence. b Fluorescent image showing the red signal of IRDye $\mathbb{8} 800 \mathrm{CW}$ EGF; green is tissue autofluorescence. c Fluorescent composite image showing both signals simultaneously (purple); green is tissue autofluorescence. d Graphic showing peak signal intensity of MMPSense ${ }^{\mathrm{TM}} 680$ at $700 \mathrm{~nm}$ (b/ue line), unmixed from autofluorescence (green line). e Graphic showing peak signal intensity of IRDye $\mathbb{R} 800 \mathrm{CW}$ EGF at $800 \mathrm{~nm}$ (red line), unmixed from autofluorescence (green line). The MMPSense signal covers a larger area compared to

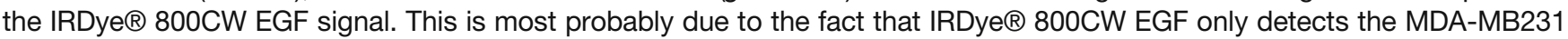
cells, whereas MMPSense also detects the highly increased osteoclastic bone resorption (ostelytic lesion) as a result of matrix metalloproteinase-9 expression of resorbing osteoclasts. AF autofluorescence.

Imaging over a range of NIR wavelengths enables correction for photon-tissue interaction and spectral unmixing of fluorescent signals. In preclinical studies, spectral unmixing has already proven to be superior to conventional fluorescence detection and was shown to greatly improve sensitivity and localization accuracy of NIR cameras [4749]. However, these systems are not able to unmix the signals in real-time, which would be required for intraoperative image-guided surgery.

Currently, two new camera systems have been developed that can detect and unmix NIR fluorescence in real-time.
The first system has been developed by the group of Ntziachristos [50], which implements a correction scheme that improves the accuracy of epi-illumination fluorescence images for light intensity variation in tissues. The implementation is based on the use of three cameras operating in parallel, utilizing a common main objective, which allows for the concurrent collection of color, fluorescence, and light attenuation images at the excitation wavelength from the same field of view. The correction is based on a ratio approach of fluorescence over light attenuation images. Color images and video are used for
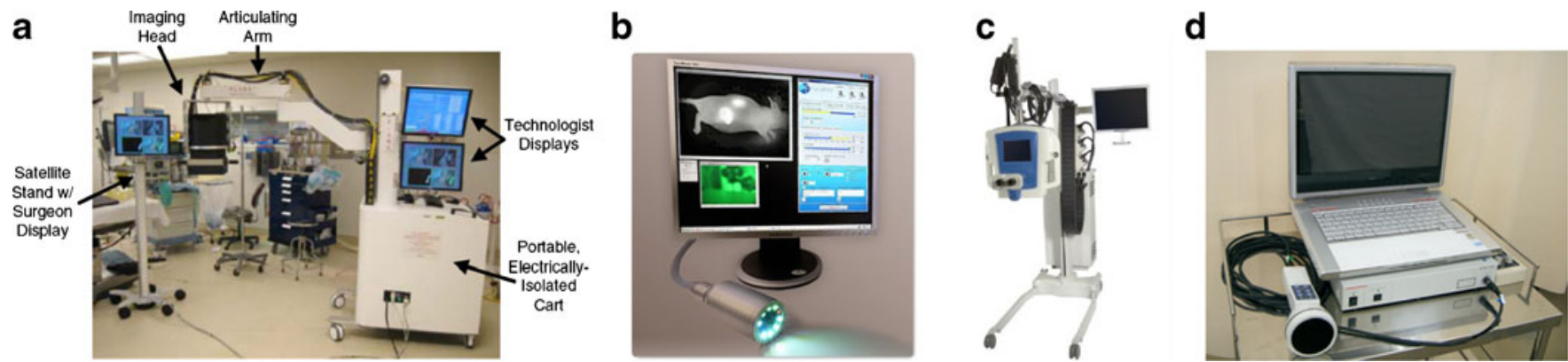

Fig. 5. Near-infrared intra-operative camera systems. a FLARETM camera system (www.frangionilab.org). b Artist impression of Fluobeam ${ }^{\mathrm{TM}}$ (www.fluoptics.com). c Artemis ${ }^{\mathrm{TM}}$ camera system (www.O2view.com). d The Photodynamic Eye (Hamamatsu Photonics, Hamamatsu City, Japan). 
surgical guidance and for registration with the corrected fluorescence images. The second camera system is the Artemis, developed by O2view (Marken, The Netherlands). The Artemis is a real-time stereoscopic imaging system that combines visible light with NIR light images. It is equipped with a laser diode and the camera system allows additional 3D visualization of fluorescent molecules, superimposed on color stereoscopic vision. It has the potential to make multispectral images because it uses a five-channel prism (covering the range from 400 $1000 \mathrm{~nm}$ ) enabling the capture of images from five different $\mathrm{CCD} / \mathrm{CMOS}$ sensors simultaneously with five different color bands. The Artemis also has a zoom function and provides real $3 \mathrm{D}$ pictures by grabbing two different images coming from two different optical axes. This new system will be tested in our institute (Leiden University Medical Center) to optimize the Artemis for intra-operative image-guided surgery (CTMM MUSIS project: www.ctmm.com)

\section{Sentinel Lymph Node Mapping}

The first steps for translation of this technique to the clinic have been made in sentinel lymph node mapping. The sentinel lymph node is the first lymph node to which the lymphatic fluid coming from the tumor drains and in which tumor cells will first metastasize. Currently, lymphatic imaging is performed using dye-injection, nuclear imaging, CT, and MRI [51], which each has their specific limitations regarding sensitivity, resolution, exposure to radioactivity, or practical use. NIR fluorescence imaging allows for high spatial and temporal resolution without ionizing radiation, making it an easy-to-use and safe technique. With parallel imaging of visible and near-infrared light, the contrast agents can be traced to the sentinel lymph nodes in real-time, without affecting the visual appearance of the surgical field.

Considering the recent clinical results using intraoperative NIR fluorescence cameras $[45,52]$ or portable NIR-imaging devices [53], sentinel lymph node mapping is one of the most promising clinical applications for NIR fluorescence imaging in the field of oncology.

\section{Other Surgical Applications}

Intra-operative optical imaging camera systems are being developed resulting in the detection of a variety of tumors in mice during surgical procedures. In addition to intraoperative tumor detection, endoscopic systems are under development for diagnostic and surgical applications [54].

In neurosurgery, the use of 5-aminolevulinic acid for detection of malignant gliomas by optical imaging techniques has been recently studied. The intra-operative use for fluorescence guidance has been described in a phase II trial as an effective adjunct in the surgery of recurrent malignant gliomas [55].

\section{Future Directions}

The use of NIR fluorescence optical imaging in the field of sentinel lymph node mapping is rapidly expanding in clinical oncology. ICG, approved by the Food and Drug Administration (FDA) for various other applications, will play a major role in this respect, potentially demonstrating the clinical advantage over current lymph node mapping protocols. However, FDA approval of organic fluorophores is a necessary step towards clinical targeting of tumor specific signals. Once these agents are also found to be safe, it is anticipated that the initial steps will be taken in conjugating them to already FDA approved and clinically used monoclonal antibodies (e.g., cetuximab, bevacizumab). In the following years, FDA approval of the activatable agents is required to fully exploit the possibilities of optical imaging, and thereby enhance the specificity and sensitivity of this technique. Furthermore, if the toxicity issues of nanoparticles can be solved, interesting developments in that field can be expected due to the aforementioned advantages regarding multimodality and multitargeted imaging. All these developments will have to be accompanied by parallel development of adequate, manageable, intra-operative camera systems.

Very recently, intriguing research regarding undetectable fluorescence of non-fluorescent, light-absorbing molecules has been described by stimulating photon emission techniques [56]. However, as yet far from practical applications, these studies hold promising possibilities for clinical or maybe even intra-operative use in the years to come.

The ultimate goal of the research that is presented in this article is intra-operative tumor imaging with optimal sensitivity and specificity. In order to achieve this, it is anticipated that a combination of different strategies that each target specific hallmarks of cancer will prove to be a key factor in guiding the oncologic surgeon towards optimal radical resection and clinical results.

Acknowledgments. This research was supported by the project of Center for Translational Molecular Medicine (CTMM) - Intra-operative Multi-Spectral Imaging Systems for Radical Tumor Resection (MUSIS). J.S.D. Mieog is a MD-medical research trainee funded by The Netherlands Organisation for Health Research and Development (grant nr. 92003526). T.J.A. Snoeks is a $\mathrm{PhD}$ student funded by The Dutch Cancer Foundation Koningin Wilhelmina Fonds (Grant No. UL2007-3801).

Conflict of interest disclosure. The authors declare that they do not have a conflict of interest.

Open Access. This article is distributed under the terms of the Creative Commons Attribution Noncommercial License which permits any noncommercial use, distribution, and reproduction in any medium, provided the original author(s) and source are credited.

\section{References}

1. Frangioni JV (2008) New technologies for human cancer imaging. J Clin Oncol 26:4012-4021

2. Hanahan D, Weinberg RA (2000) The hallmarks of cancer. Cell 100:57-70

3. Semenza GL, Artemov D, Bedi A et al (2001) 'The metabolism of tumours': 70 years later. Novartis Found Symp 240:251-260

4. Kelloff GJ, Hoffman JM, Johnson B et al (2005) Progress and promise of FDG-PET imaging for cancer patient management and oncologic drug development. Clin Cancer Res 11:2785-2808 
5. Strong VE, Humm J, Russo P et al (2008) A novel method to localize antibody-targeted cancer deposits intraoperatively using handheld PET beta and gamma probes. Surg Endosc 22:386-391

6. de Jong BW, Schut TC, Maquelin K et al (2006) Discrimination between nontumor bladder tissue and tumor by Raman spectroscopy. Anal Chem 78:7761-7769

7. Shetty G, Kendall C, Shepherd N, Stone N, Barr H (2006) Raman spectroscopy: elucidation of biochemical changes in carcinogenesis of oesophagus. Br J Cancer 94:1460-1464

8. Stone N, Stavroulaki P, Kendall C, Birchall M, Barr H (2000) Raman spectroscopy for early detection of laryngeal malignancy: preliminary results. Laryngoscope 110:1756-1763

9. Nijssen A, Koljenovic S, Bakker Schut TC, Caspers PJ, Puppels GJ (2009) Towards oncological application of Raman spectroscopy. J Biophotonics 2:29-36

10. DaCosta RS, Andersson H, Wilson BC (2003) Molecular fluorescence excitation-emission matrices relevant to tissue spectroscopy. Photochem Photobiol 78:384-392

11. Georgakoudi I, Jacobson BC, Muller MG et al (2002) NAD(P)H and collagen as in vivo quantitative fluorescent biomarkers of epithelial precancerous changes. Cancer Res 62:682-687

12. Lane PM, Gilhuly T, Whitehead P et al (2006) Simple device for the direct visualization of oral cavity tissue fluorescence. J Biomed Opt 11:024006

13. Lee P, van den Berg RM, Lam S et al (2009) Color fluorescence ratio for detection of bronchial dysplasia and carcinoma In situ. Clin Cancer Res 15:4700-4705

14. Roblyer D, Richards-Kortum R, Sokolov K et al (2008) Multispectral optical imaging device for in vivo detection of oral neoplasia. J Biomed Opt 13:024019

15. Alexander S, Koehl GE, Hirschberg M, Geissler EK, Friedl P (2008) Dynamic imaging of cancer growth and invasion: a modified skin-fold chamber model. Histochem Cell Biol 130:1147-1154

16. de Veld DC, Witjes MJ, Sterenborg HJ, Roodenburg JL (2005) The status of in vivo autofluorescence spectroscopy and imaging for oral oncology. Oral Oncol 41:117-131

17. Nioka S, Chance B (2005) NIR spectroscopic detection of breast cancer. Technol Cancer Res Treat 4:497-512

18. Choe R, Konecky SD, Corlu A et al (2009) Differentiation of benign and malignant breast tumors by in vivo three-dimensional parallel-plate diffuse optical tomography. J Biomed Opt 14:024020

19. Intes $X$ (2005) Time-domain optical mammography SoftScan: initial results. Acad Radiol 12:934-947

20. Pierce MC, Javier DJ, Richards-Kortum R (2008) Optical contrast agents and imaging systems for detection and diagnosis of cancer. Int $\mathrm{J}$ Cancer 123:1979-1990

21. Aoki T, Yasuda D, Shimizu Y et al (2008) Image-guided liver mapping using fluorescence navigation system with indocyanine green for anatomical hepatic resection. World J Surg 32:1763-1767

22. Ishizawa T, Fukushima N, Shibahara J et al (2009) Real-time identification of liver cancers by using indocyanine green fluorescent imaging. Cancer 115:2491-2504

23. Mahmood U, Weissleder R (2003) Near-infrared optical imaging of proteases in cancer. Mol Cancer Ther 2:489-496

24. Wunderbaldinger P, Turetschek K, Bremer C (2003) Near-infrared fluorescence imaging of lymph nodes using a new enzyme sensing activatable macromolecular optical probe. Eur Radiol 13:2206-2211

25. Jiang T, Olson ES, Nguyen QT, Roy M, Jennings PA, Tsien RY (2004) Tumor imaging by means of proteolytic activation of cell-penetrating peptides. Proc Natl Acad Sci U S A 101:17867-17872

26. Wunder A, Straub RH, Gay S, Funk J, Muller-Ladner U (2005) Molecular imaging: novel tools in visualizing rheumatoid arthritis. Rheumatology (Oxford) 44:1341-1349

27. Klohs J, Baeva N, Steinbrink J et al (2009) In vivo near-infrared fluorescence imaging of matrix metalloproteinase activity after cerebral ischemia. J Cereb Blood Flow Metab 29:1284-1292

28. Gleysteen JP, Duncan RD, Magnuson JS, Skipper JB, Zinn K, Rosenthal EL (2007) Fluorescently labeled cetuximab to evaluate head and neck cancer response to treatment. Cancer Biol Ther 6:1181-1185

29. Lee SB, Hassan M, Fisher R et al (2008) Affibody molecules for in vivo characterization of HER2-positive tumors by near-infrared imaging. Clin Cancer Res 14:3840-3849

30. Withrow KP, Newman JR, Skipper JB et al (2008) Assessment of bevacizumab conjugated to Cy5.5 for detection of head and neck cancer xenografts. Technol Cancer Res Treat 7:61-66
31. Ogawa M, Kosaka N, Longmire MR, Urano Y, Choyke PL, Kobayashi H (2009) Fluorophore-quencher based activatable targeted optical probes for detecting in vivo cancer metastases. Mol Pharm 6(2):386-395

32. Backer MV, Levashova Z, Patel V et al (2007) Molecular imaging of VEGF receptors in angiogenic vasculature with single-chain VEGFbased probes. Nat Med 13:504-509

33. Kovar JL, Volcheck WM, Chen J, Simpson MA (2007) Purification method directly influences effectiveness of an epidermal growth factorcoupled targeting agent for noninvasive tumor detection in mice. Anal Biochem 361:47-54

34. Adams KE, Ke S, Kwon S et al (2007) Comparison of visible and nearinfrared wavelength-excitable fluorescent dyes for molecular imaging of cancer. J Biomed Opt 12:024017

35. Chen K, Xie J, Chen X (2009) RGD-human serum albumin conjugates as efficient tumor targeting probes. Mol Imaging 8:65-73

36. Jin ZH, Razkin J, Josserand V et al (2007) In vivo noninvasive optical imaging of receptor-mediated RGD internalization using self-quenched Cy5-labeled RAFT-c(-RGDfK-)(4). Mol Imaging 6:43-55

37. Kossodo S, Pickarski M, Lin SA et al (2009) Dual in vivo quantification of integrin-targeted and protease-activated agents in cancer using fluorescence molecular tomography (FMT). Mol Imaging Biol (in press)

38. Zhou H, Luby-Phelps K, Mickey BE, Habib AA, Mason RP, Zhao D (2009) Dynamic near-infrared optical imaging of 2-deoxyglucose uptake by intracranial glioma of athymic mice. PLoS ONE 4:e8051

39. Mulder WJ, Castermans K, van Beijnum JR et al (2009) Molecular imaging of tumor angiogenesis using alphavbeta3-integrin targeted multimodal quantum dots. Angiogenesis 12:17-24

40. Kirchner C, Liedl T, Kudera S et al (2005) Cytotoxicity of colloidal CdSe and CdSe/ZnS nanoparticles. Nano Lett 5:331-338

41. Lewinski N, Colvin V, Drezek R (2008) Cytotoxicity of nanoparticles. Small 4:26-49

42. Choi J, Burns AA, Williams RM et al (2007) Core-shell silica nanoparticles as fluorescent labels for nanomedicine. J Biomed Opt 12:064007

43. Xie G, Sun J, Zhong G, Shi L, Zhang D (2009) Biodistribution and toxicity of intravenously administered silica nanoparticles in mice. Arch Toxicol 84(3):183-190

44. Oberdorster G, Oberdorster E, Oberdorster J (2005) Nanotoxicology: an emerging discipline evolving from studies of ultrafine particles. Environ Health Perspect 113:823-839

45. De Grand AM, Frangioni JV (2003) An operational near-infrared fluorescence imaging system prototype for large animal surgery. Technol Cancer Res Treat 2:553-562

46. Stockdale A, Oketokoun R, Gioux S, Frangioni JV (2010) MiniFLARE: a compact and ergonomic dual-channel near-infrared fluorescence image-guided surgery system (Abstract)

47. Mansfield JR, Hoyt C, Levenson RM (2008) Visualization of microscopy-based spectral imaging data from multi-label tissue sections. Curr Protoc Mol Biol Chapter 14: Unit

48. Mayes P, Dicker D, Liu Y, El-Deiry W (2008) Noninvasive vascular imaging in fluorescent tumors using multispectral unmixing. Biotechniques $45: 459-464$

49. Xu H, Rice BW (2009) In vivo fluorescence imaging with a multivariate curve resolution spectral unmixing technique. J Biomed Opt 14:064011

50. Themelis G, Yoo JS, Soh KS, Schulz R, Ntziachristos V (2009) Realtime intraoperative fluorescence imaging system using light-absorption correction. J Biomed Opt 14:064012

51. Barrett T, Choyke PL, Kobayashi H (2006) Imaging of the lymphatic system: new horizons. Contrast Media Mol Imaging 1:230-245

52. Troyan SL, Kianzad V, Gibbs-Strauss SL et al (2009) The FLARE intraoperative near-infrared fluorescence imaging system: a first-inhuman clinical trial in breast cancer sentinel lymph node mapping. Ann Surg Oncol 16:2943-2952

53. Tagaya N, Yamazaki R, Nakagawa A et al (2008) Intraoperative identification of sentinel lymph nodes by near-infrared fluorescence imaging in patients with breast cancer. Am J Surg 195:850-853

54. Figueiredo JL, Alencar H, Weissleder R, Mahmood U (2006) Near infrared thoracoscopy of tumoral protease activity for improved detection of peripheral lung cancer. Int J Cancer 118:2672-2677

55. Nabavi A, Thurm H, Zountsas B et al (2009) Five-aminolevulinic acid for fluorescence-guided resection of recurrent malignant gliomas: a phase II study. Neurosurgery 65:1070-1076

56. Min W, Lu S, Chong S, Roy R, Holtom GR, Xie XS (2009) Imaging chromophores with undetectable fluorescence by stimulated emission microscopy. Nature 461:1105-1109 